\title{
Fermilab
}

\section{Advanced Proton Booster Design (Integrable Optics for High Intensity Beams)}

Jeffrey Eldred, Sasha Valishev

UChicago Workshop Nonlinear \& Collective Effects 28 Oct 2017 


\section{Fermilab Proton Accelerator Facility}

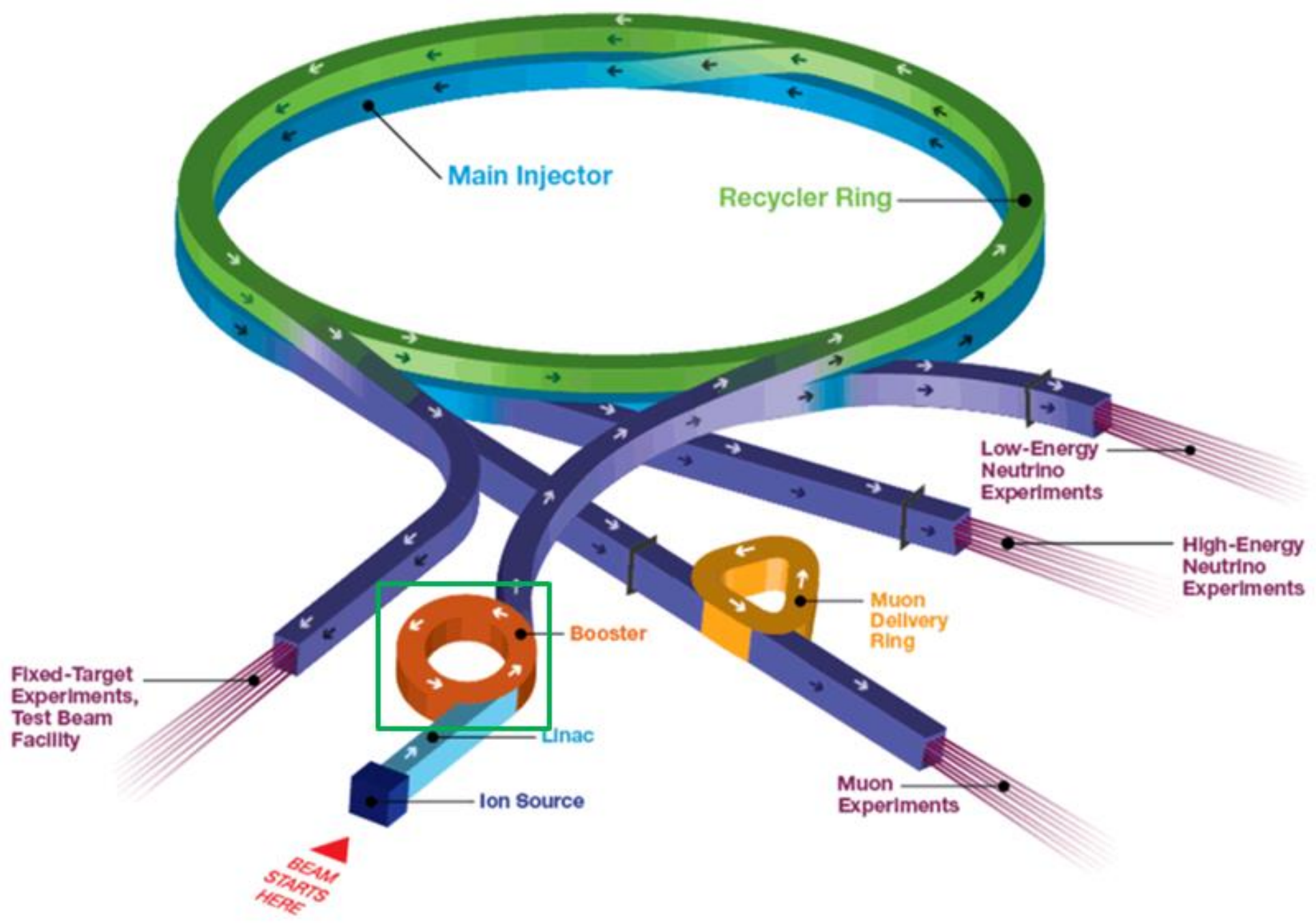

\section{荤 Fermilab}

2 Jeffrey Eldred | An RCS with Integrable Optics for the Fermilab PIP-III Upgrade

$4 / 18 / 2019$ 


\section{(Proposed) PIP-III Intensity Upgrade}

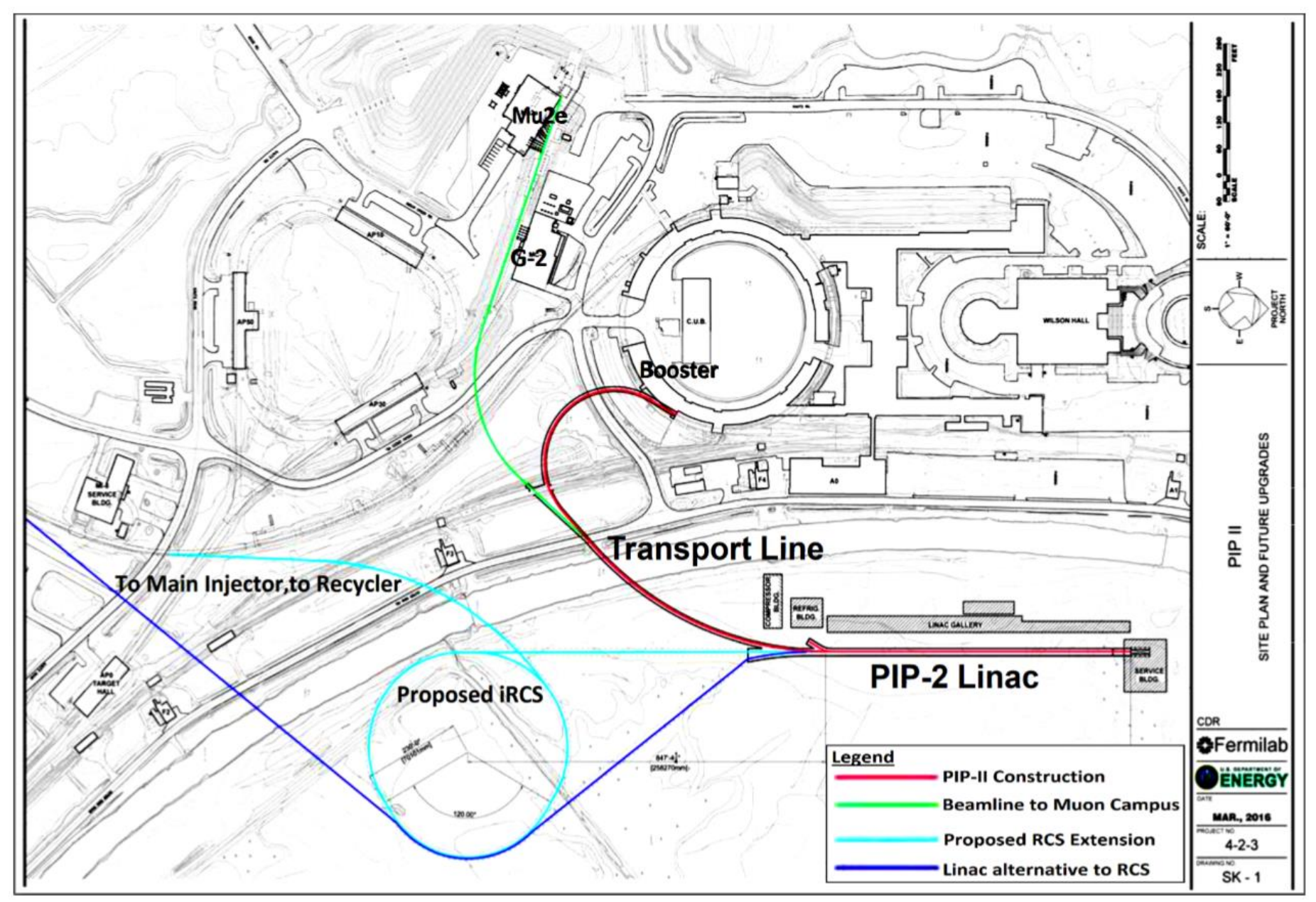

\section{芓 Fermilab}

3 Jeffrey Eldred | An RCS with Integrable Optics for the Fermilab PIP-III Upgrade 


\section{Rapid-Cycling Synchrotron Design}

- Modern RCS design calls for several features:

- Dispersion-free drifts, for RF

- Low momentum compaction factor, to avoid transition.

- Low beta functions, for maximum emittance per aperture.

- Mitigation of collective instabilities, throughout the ramp.

- Integrable optics design is a promising innovation to provide significant nonlinear focusing with two invariants of motions.

- But first, it is necessary to demonstrate integrable optics is still helpful in the presence of strong space-charge forces. 


\section{Danilov-Nagaitsev Integral Design}

Time-independent kick:

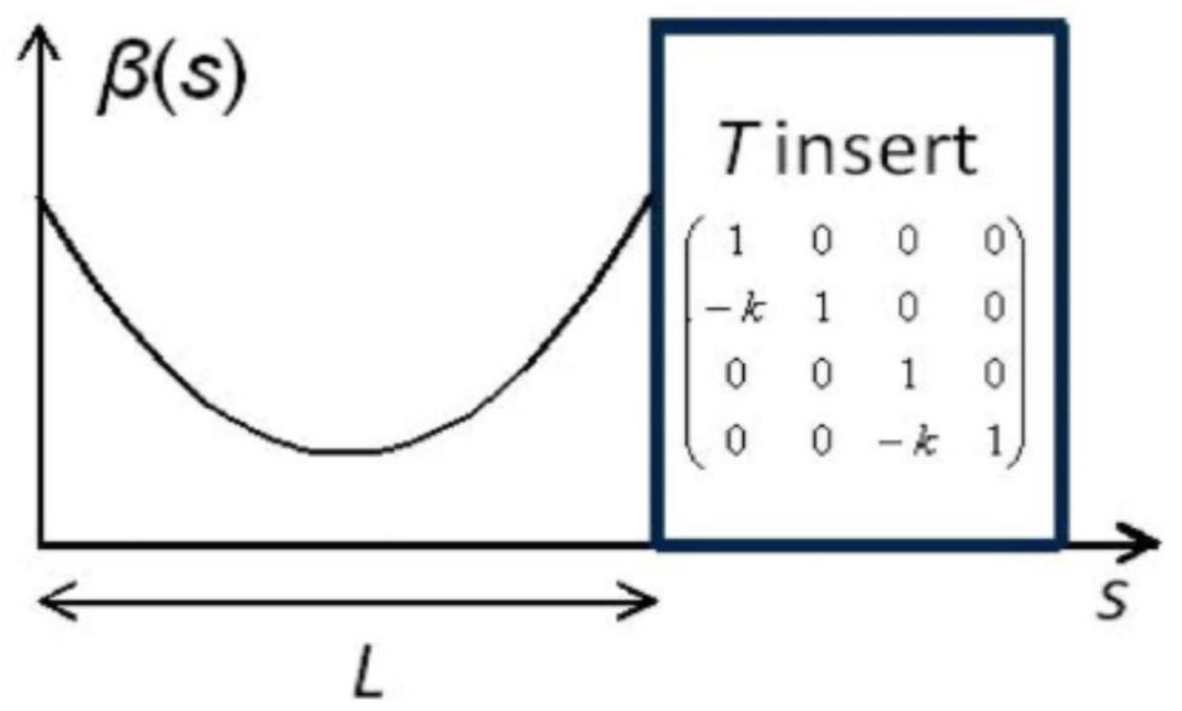

NL Insert
Nonlinear kick separable in elliptic coordinates:

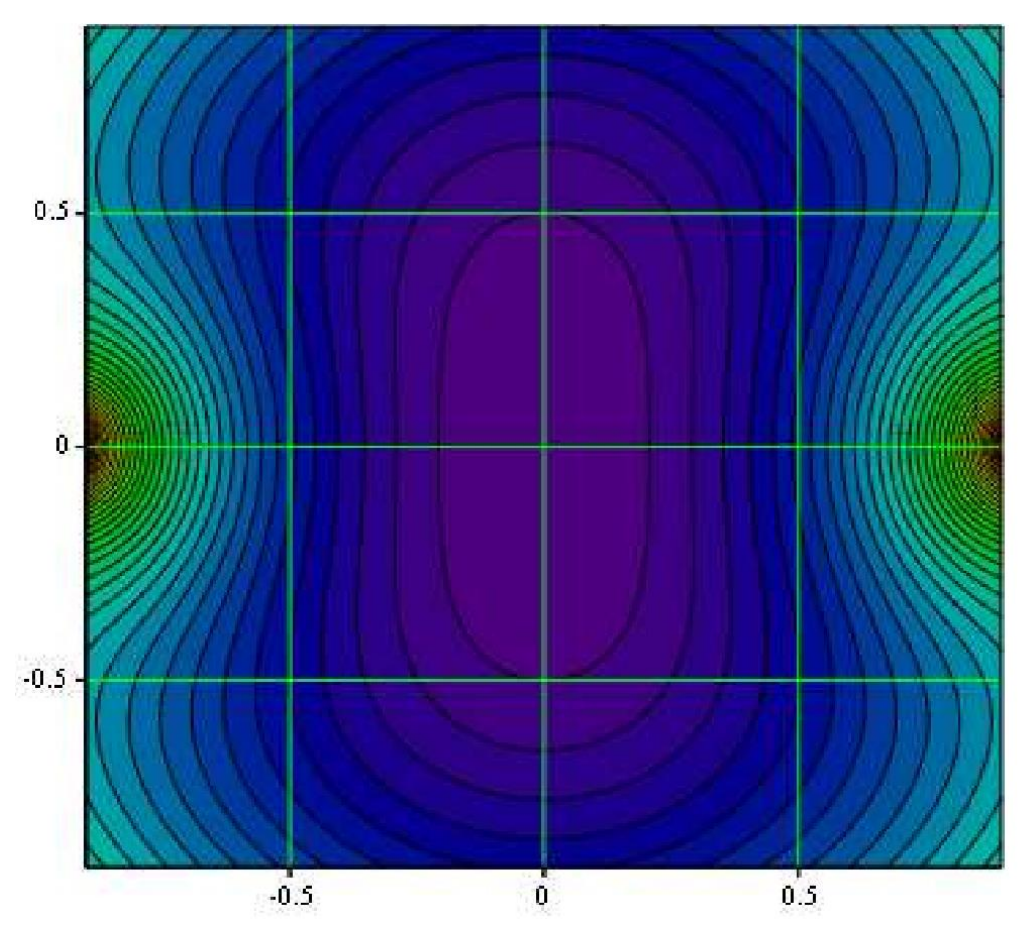

V. Danilov, S. Nagaitsev "Nonlinear Lattices with One or Two Analytic Invariants" PRST-AB 2010. 


\section{Integral Design with Periodicity}

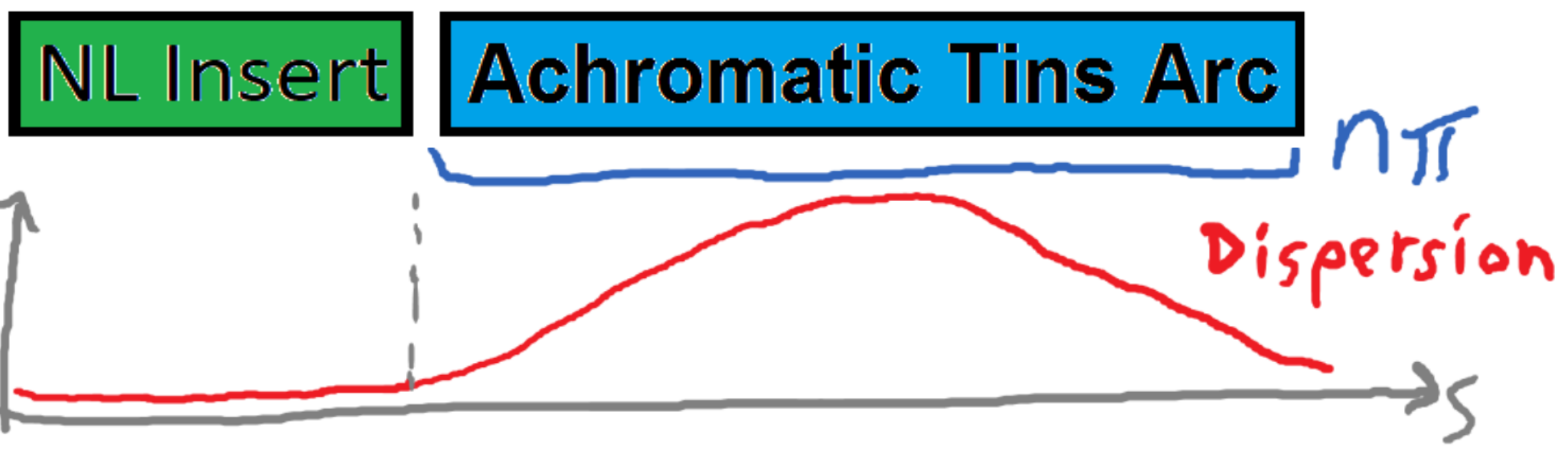

Multiple Periodic Cells:
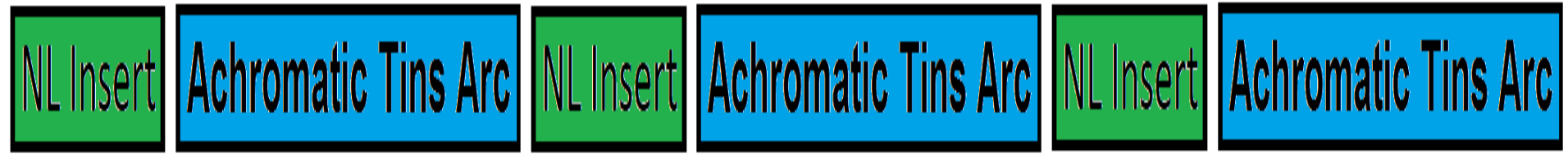

... 


\section{iRCS v2 Lattice Parameters}

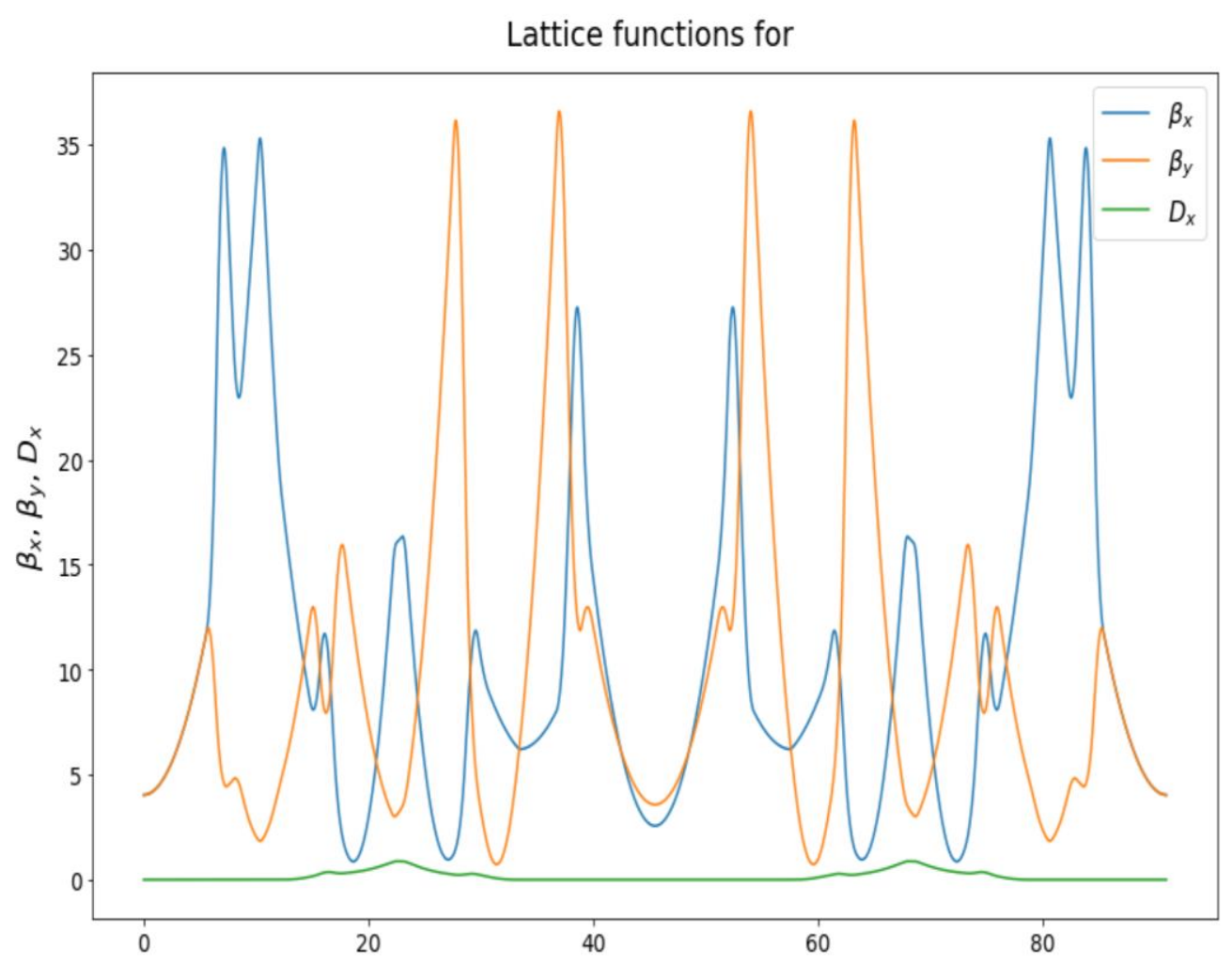

Periodicity: 6

Circumference: $\mathbf{5 4 2} \mathbf{~ m}$ Bend-radius rho: $15.4 \mathrm{~m}$ Max Beta function: $35 \mathrm{~m}$ Insertion length: $\mathbf{1 1 . 2} \mathbf{~ m}$ Betatron Tune: $\mathbf{1 6 . 8}$

Insert Phase-Advance: $\mathbf{0 . 3}$

Nonlinear t-value: $\mathbf{0 . 1 5}$ Minimum c-value: $3 \mathrm{~cm}$ Beta at insert center: $\mathbf{4} \mathbf{~ m}$ Corrected Chromaticity: $\mathbf{- 7 . 7}$ Natural Chromaticity: $\mathbf{- 3 3}$ Second-order Chromaticity: -132

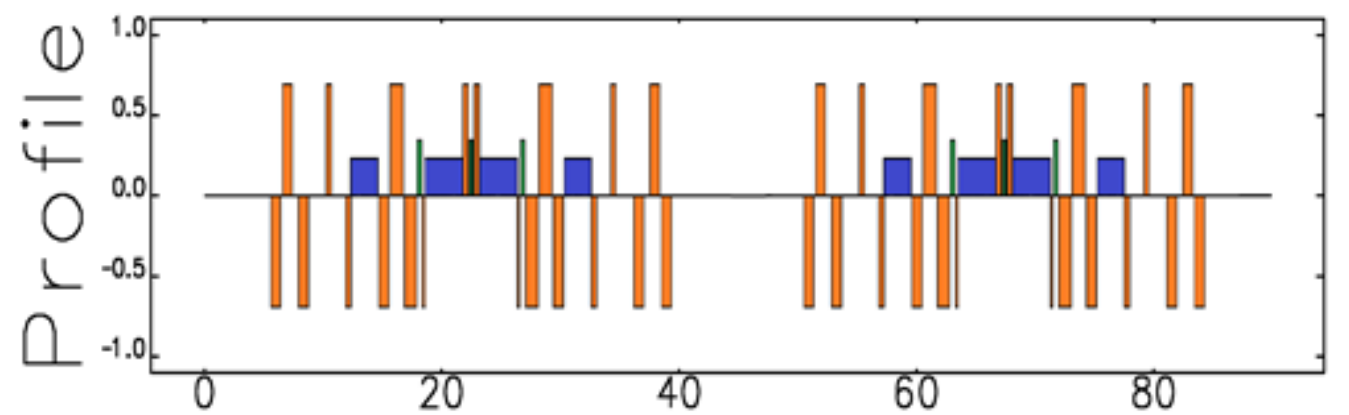




\section{Synergia Simulation of Halo formed by Beam Mismatched}




\section{Simulation Parameters}

- Coasting proton beam with 3D PIC space-charge.

- Injection energy of $0.8 \mathrm{GeV}$.

- Initial normalized emittance $20 \mathrm{~mm}$ mrad.

- 2D Waterbag beam initially distributed along equipotential contours except with a $20 \%$ mismatch.

- 500 revolutions of iRCS (3000 iterations of periodic cell).

- Phase advance through insert $\mathrm{d} \Phi=0.3$

- Nonlinear strength parameter $t=0.3$

- 1 Conventional Design, Low Intensity Beam $(\mathrm{dQ}=-0.05)$

- 2 Integrable Design, Low Intensity Beam $(\mathrm{dQ}=-0.05)$

- 3 Conventional Design, High Intensity Beam $(\mathrm{dQ}=-0.20)$

- 4 Integrable Design, High Intensity Beam $(d Q=-0.20)$ 


\section{RMS Beam Size}

\section{Conventional, Low Int.}

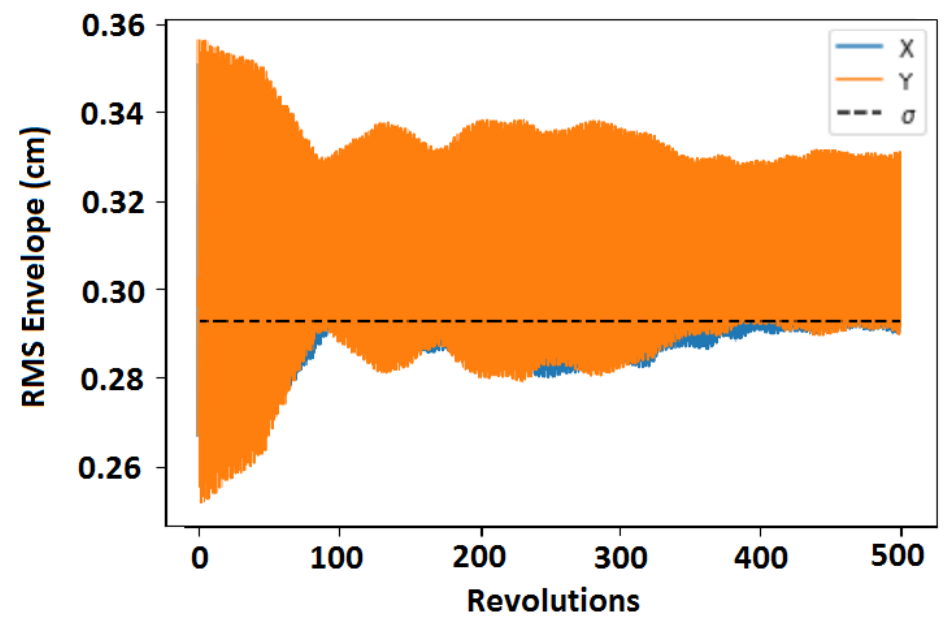

\section{Conventional, High Int.}

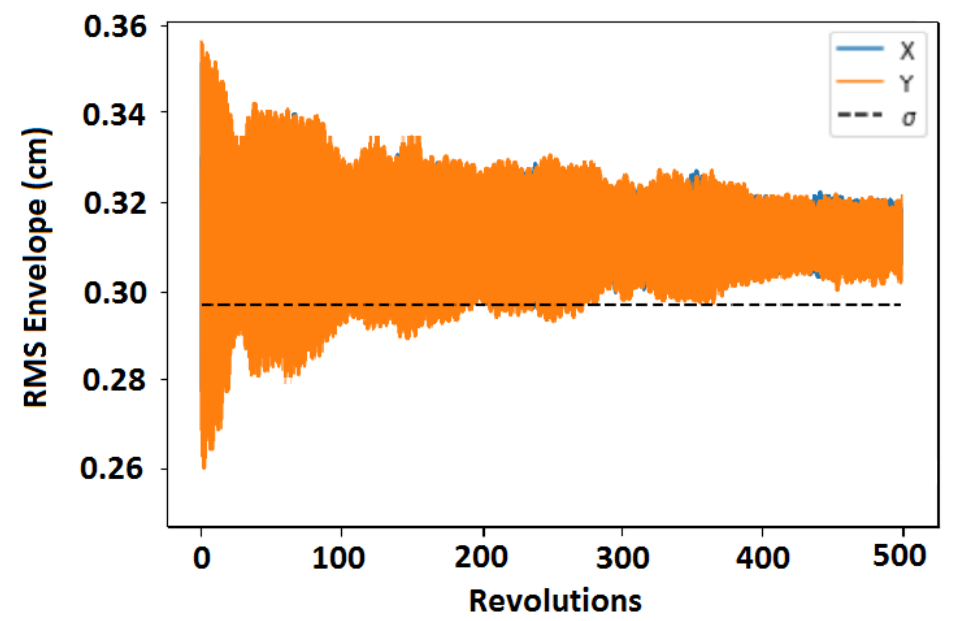

2 Integrable, Low Int.

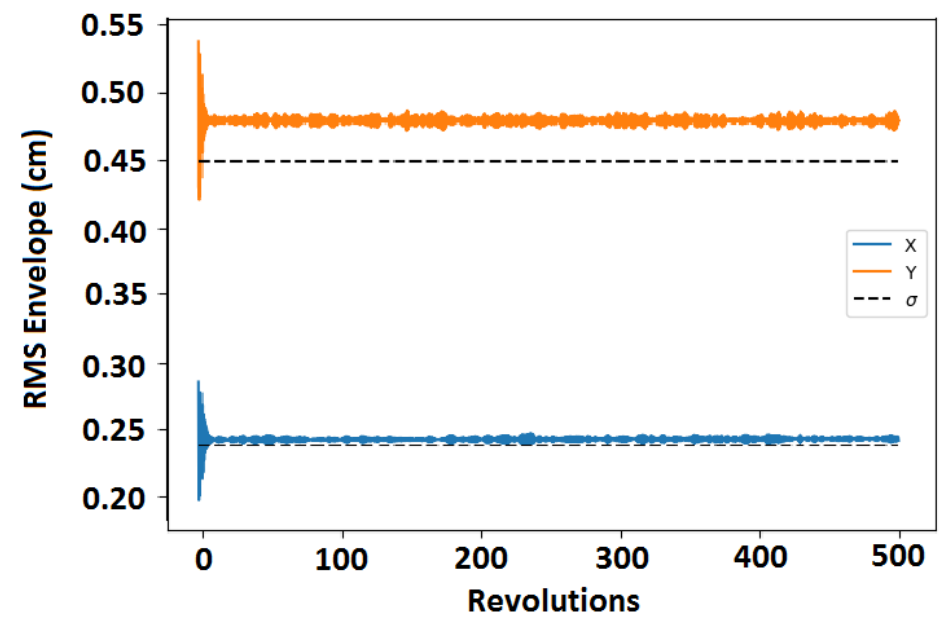

4 Integrable, High Int.

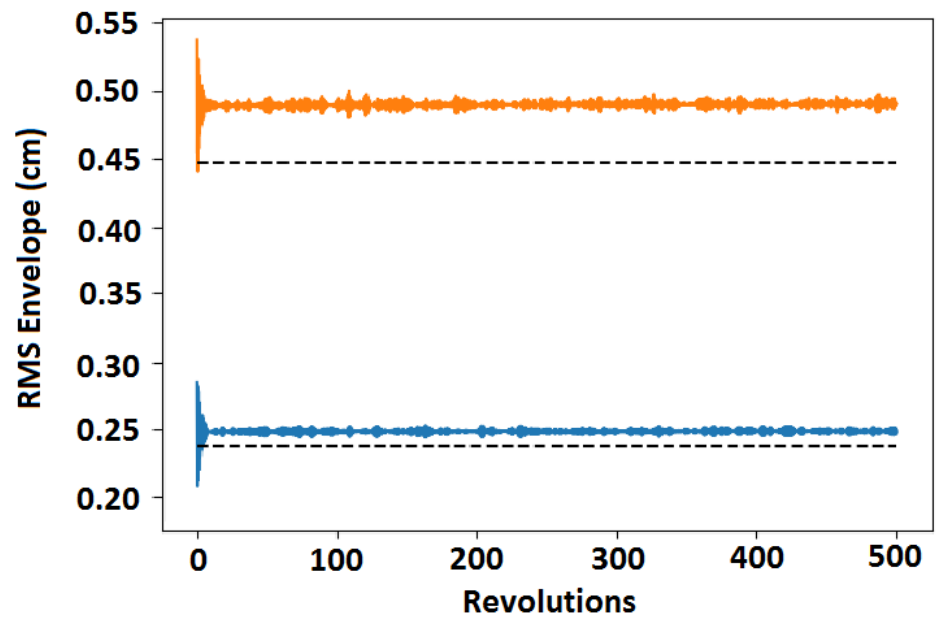

10 Jeffrey Eldred | An RCS with Integrable Optics for the Fermilab PIP-III Upgrade

$4 / 18 / 2019$ 


\section{Transverse Beam Halo}

\section{Conventional, Low Int.}

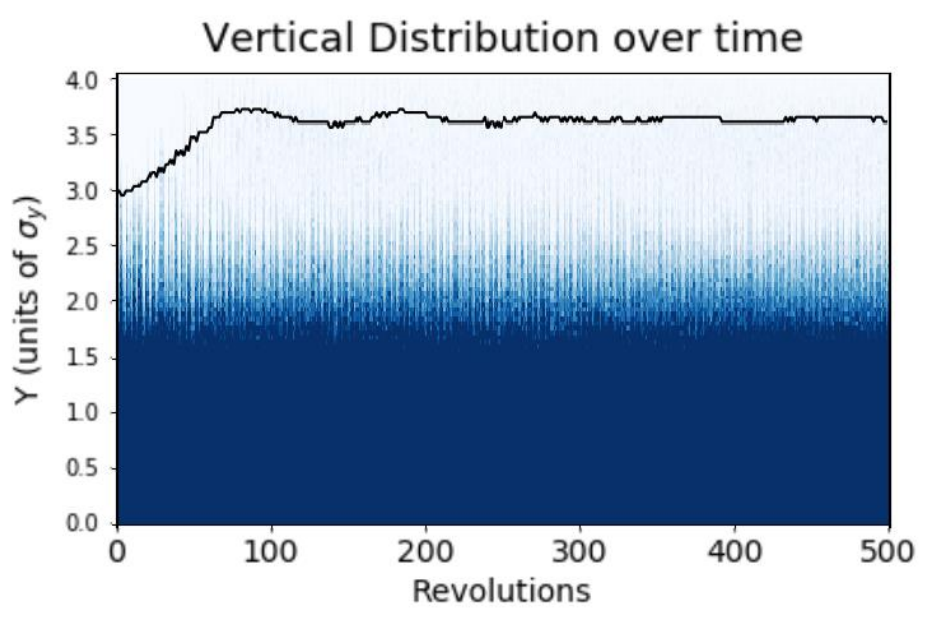

3 Conventional, High Int.

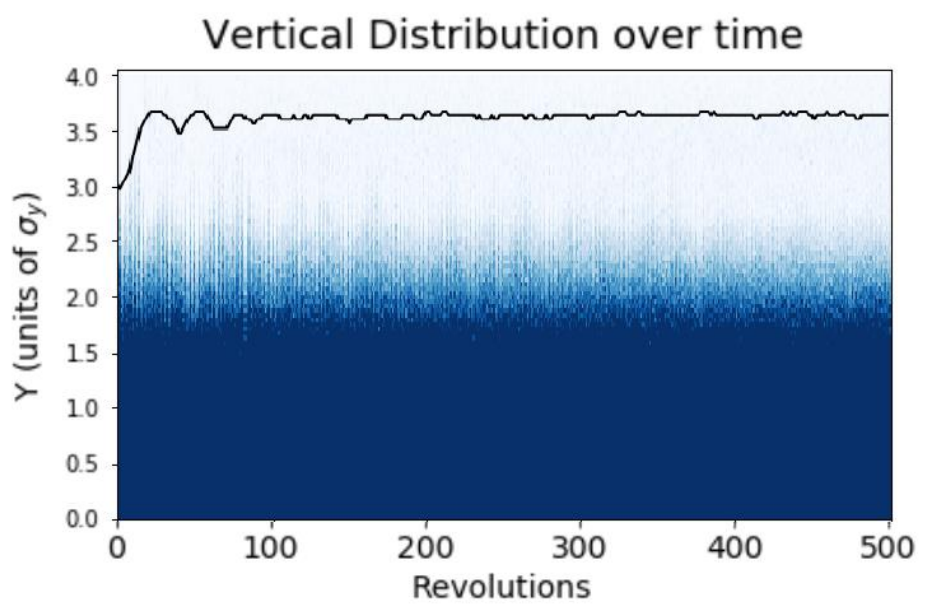

\section{Integrable, Low Int.}

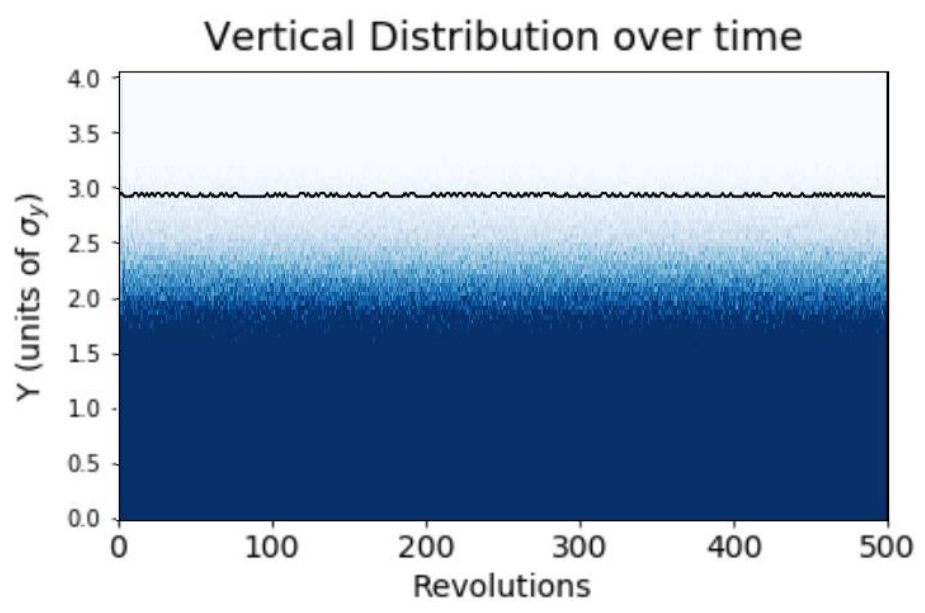

4 Integrable, High Int.

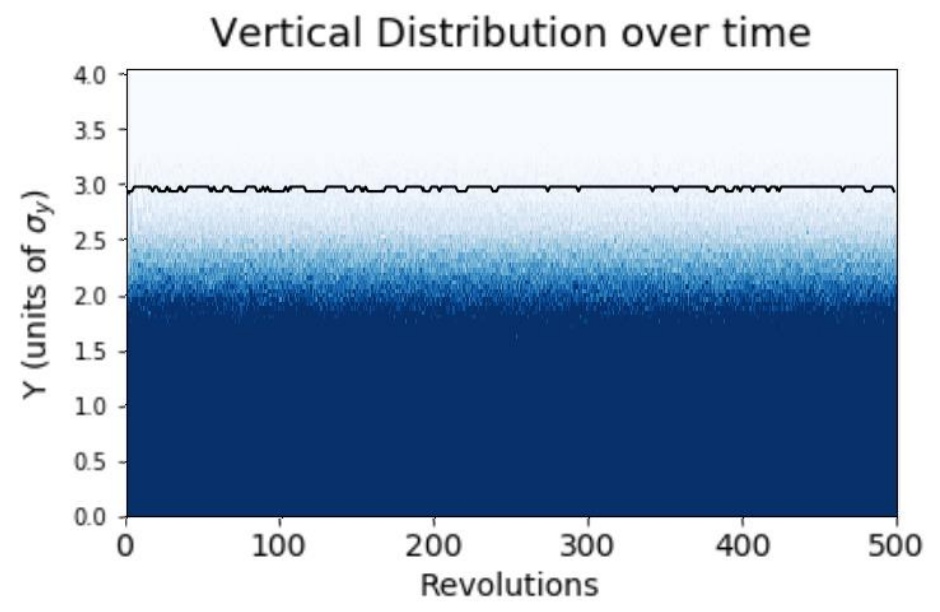




\section{Cell Betatron Tune Distribution}

\section{Conventional, Low Int.}

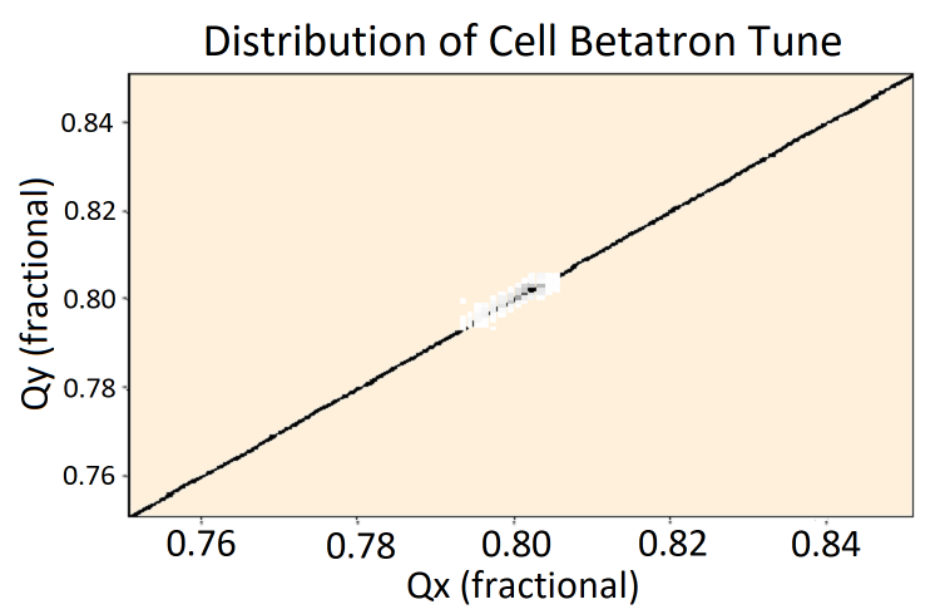

3 Conventional, High Int.

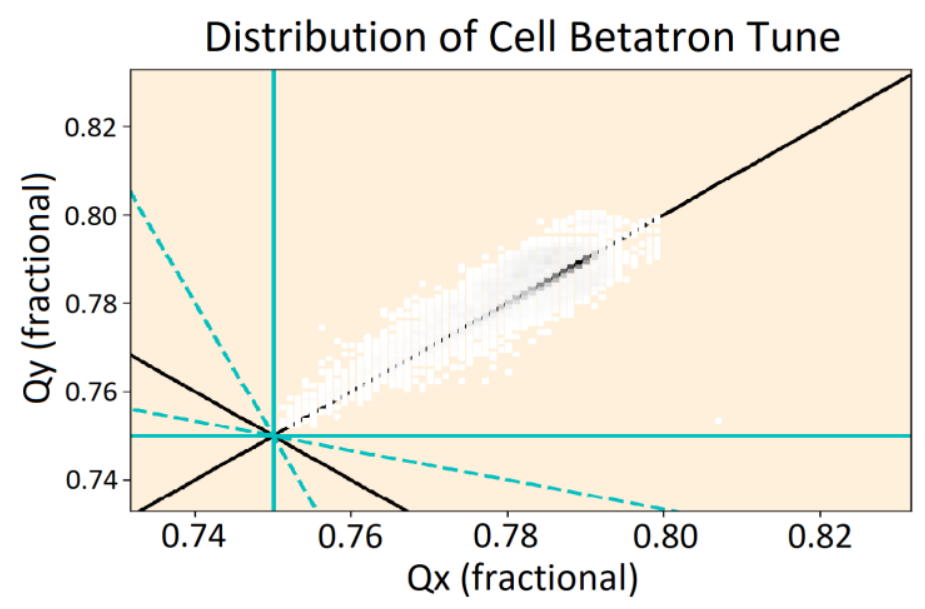

\section{Integrable, Low Int.}

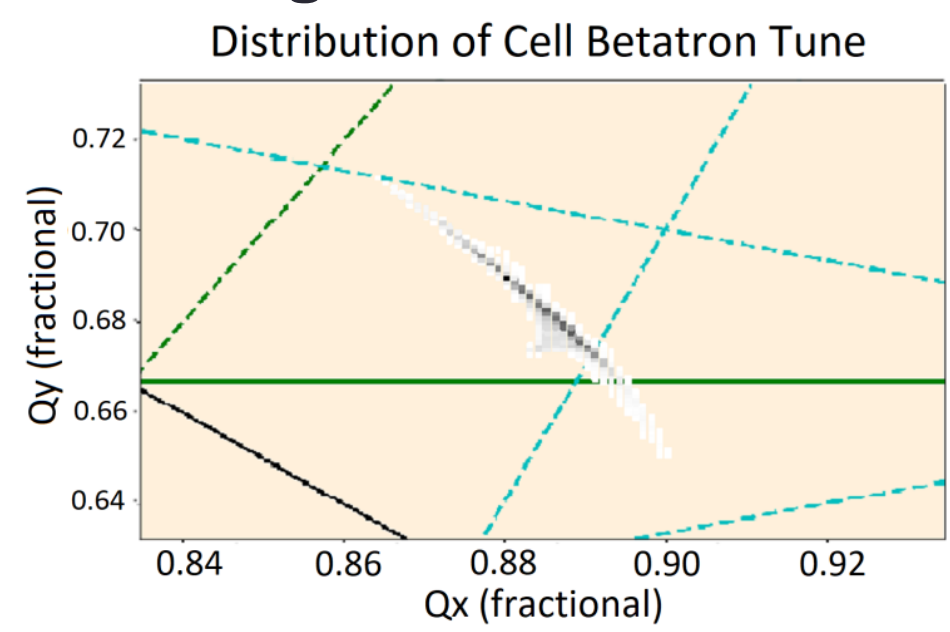

4 Integrable, High Int.

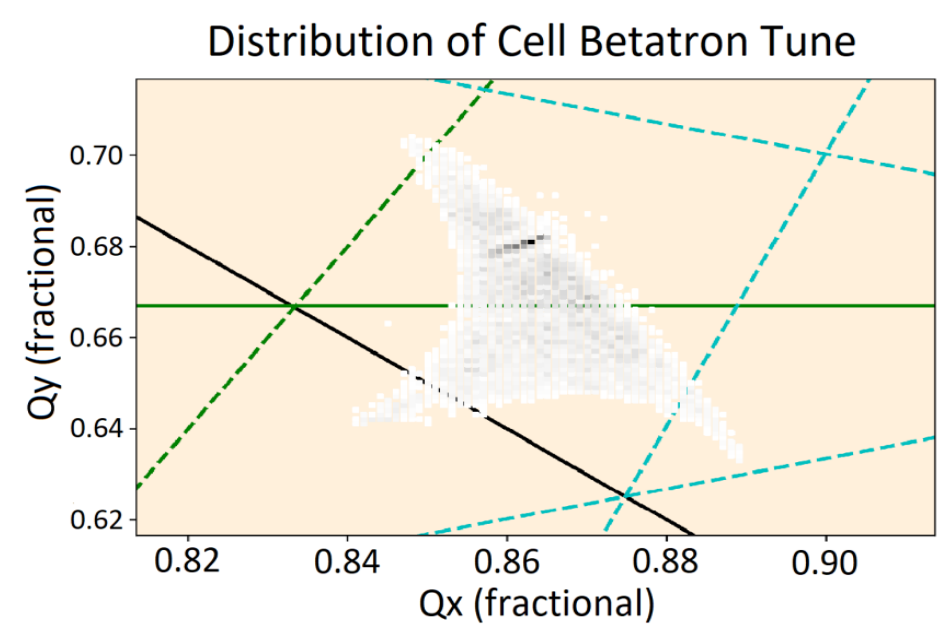




\section{Ring Betatron Tune Distribution}

\begin{tabular}{|l|c|c|}
\hline Description & $\begin{array}{c}90 \% \text { Horizontal } \\
\text { Tune Spread }\end{array}$ & $\begin{array}{c}90 \% \text { Vertical } \\
\text { Tune Spread }\end{array}$ \\
\hline 1 Conventional, Low Int. & 0.044 & 0.044 \\
\hline I Integrable, Low Int. & $\mathbf{0 . 1 2}$ & $\mathbf{0 . 1 8}$ \\
\hline 3 Conventional, High Int. & 0.16 & 0.16 \\
\hline 4 Integrable, High Int. & $\mathbf{0 . 1 5}$ & $\mathbf{0 . 2 5}$ \\
\hline
\end{tabular}

\section{Nonlinear Parameters}

Space-charge tune shift $d Q=-0.05,-0.20$

Phase advance through Insert $\mathrm{d} \Phi=0.3$

Nonlinear strength parameter $\mathbf{t}=\mathbf{0 . 3}$ 


\section{Mismatched Waterbag with Chromatic Tune Shift}




\section{Simulation Parameters}

- Chromaticity doesn't have to be zero, it just has to be matched between horizontal and vertical.

- Webb, Bruhwiler, Valishev, Nagaitsev, Danilov "Chromatic and Dispersive Effects in Nonlinear Integrable Optics" PR-AB (Submitted).

- Nominal momentum spread based on zero-charge longitudinal simulation of RF capture of injected beam.

- Chromaticity (matched horiz. \& vert.): -33

- Chromatic tune-shift (nominal): $0.25 \%$

- 2 Integrable Design, Low Intensity, $\sigma p=0$ (Monoenergetic)

- 5 Integrable Design, Low Intensity, $\sigma p=0.250 \%$ (Nominal) 


\section{RMS Beam Size}

2 Integrable WB, $\sigma p=0$

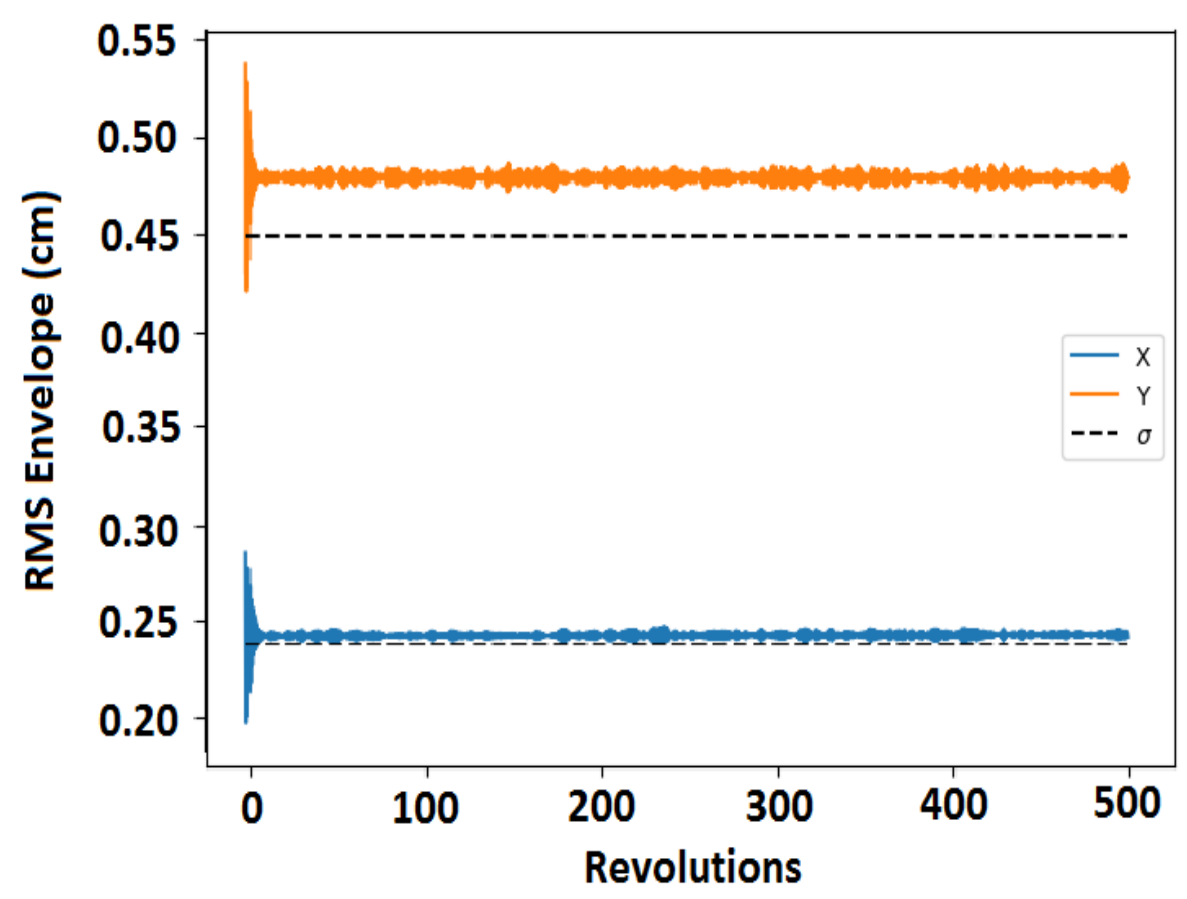

\section{Integrable WB, x20 $\sigma p$}

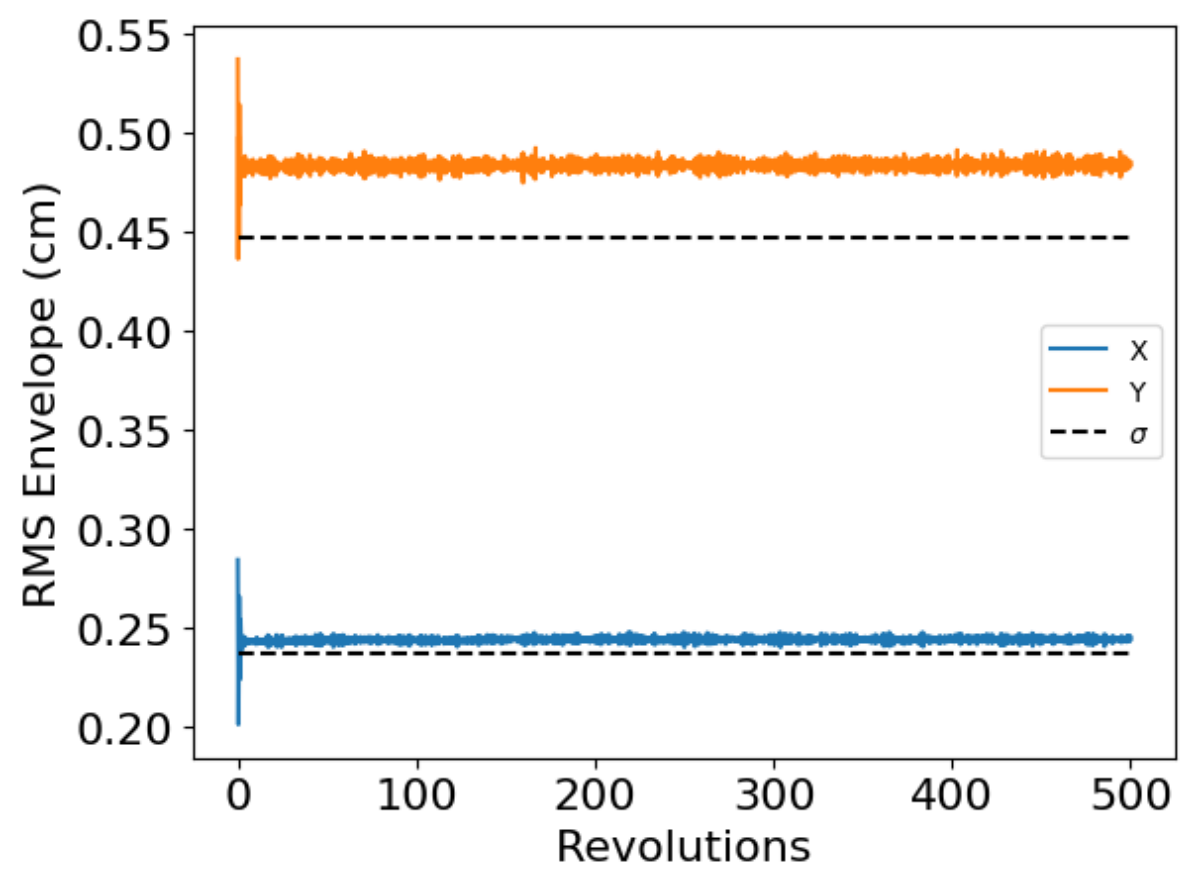




\section{Transverse Beam Halo}

2 Integrable WB, $\sigma p=0$

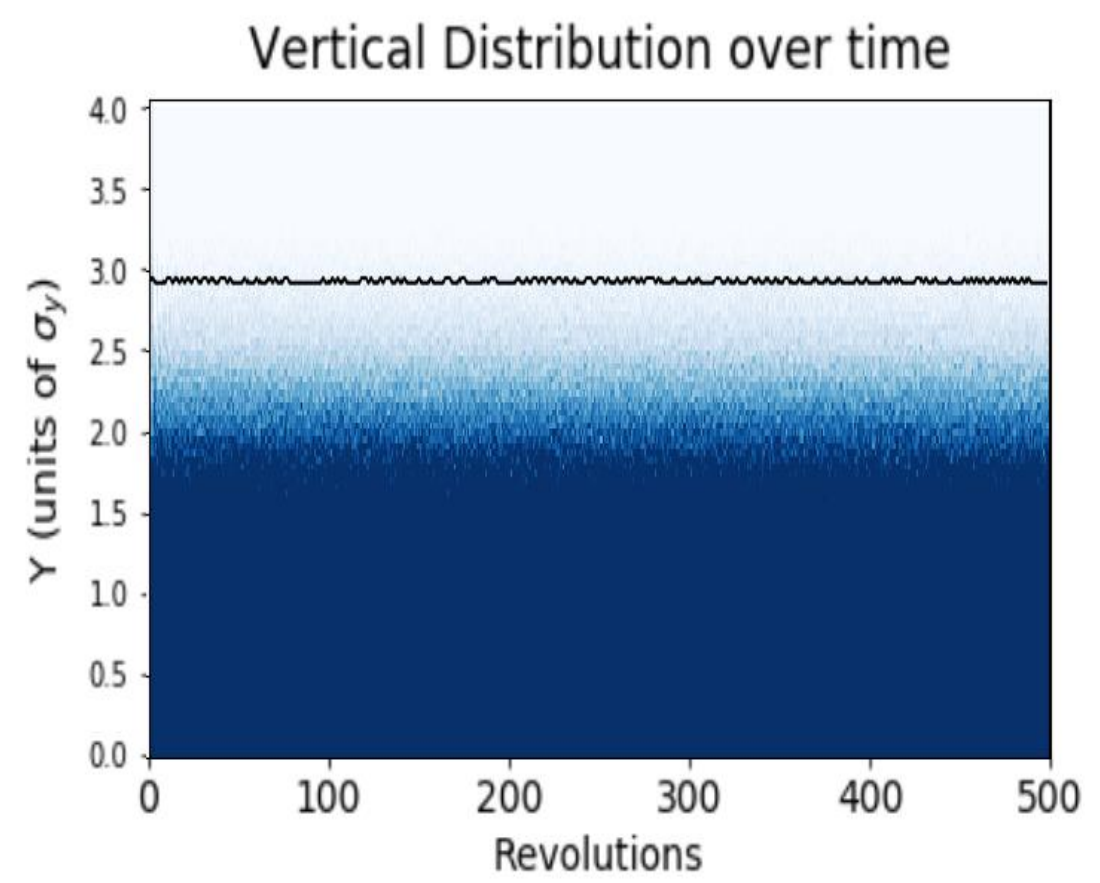

5 Integrable WB, Nominal $\sigma p$

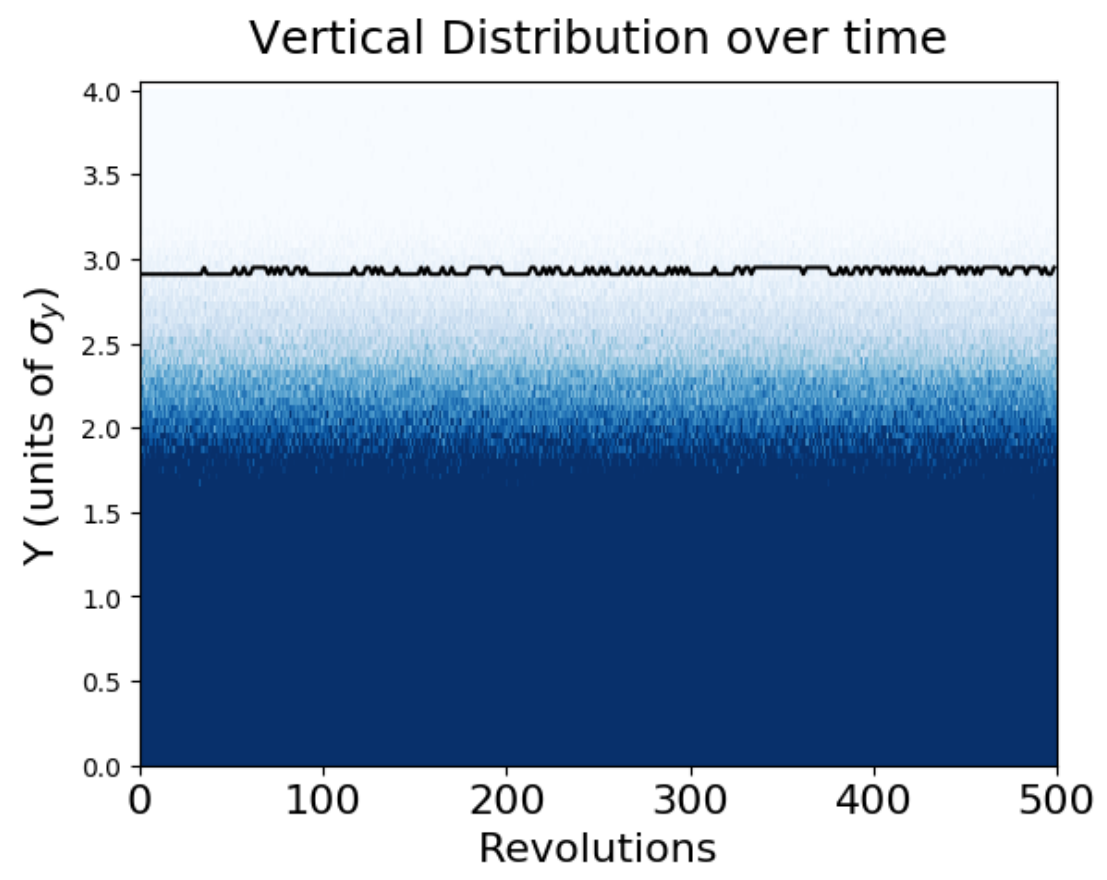




\section{Cell Betatron Tune Distribution}

2 Integrable WB, $\sigma p=0$

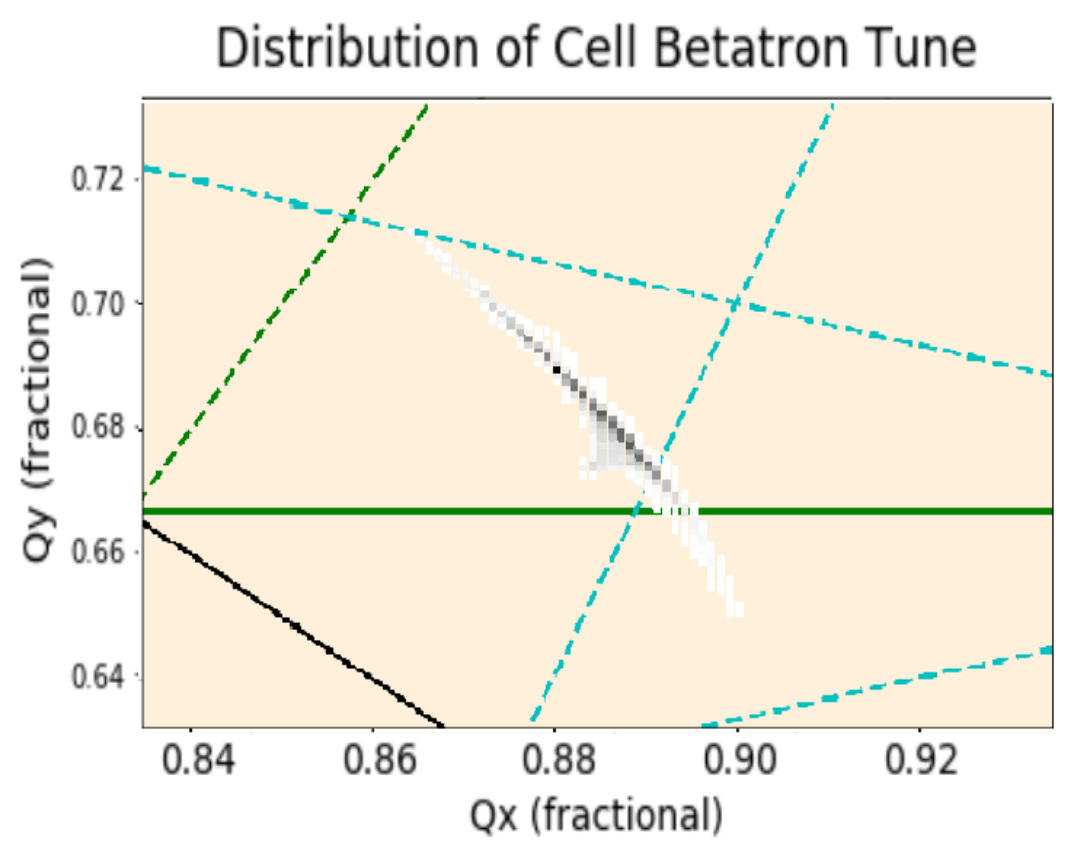

\section{Integrable WB, Nominal op}

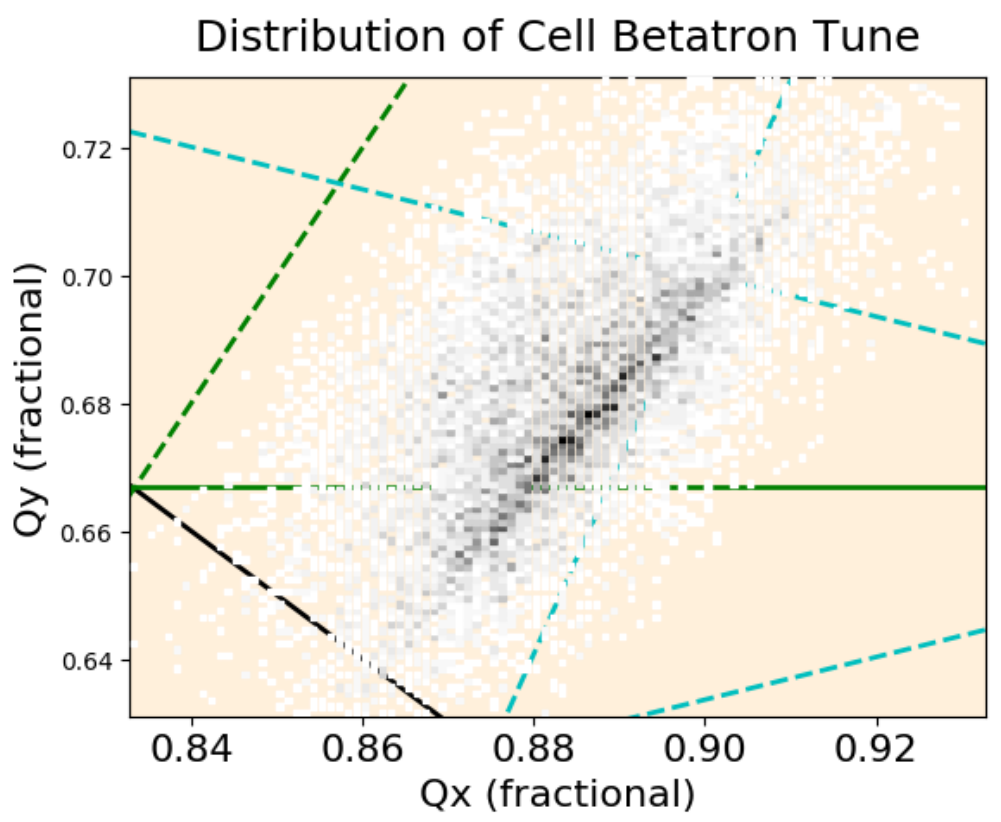

The chromatic tune-shift occurs diagonally and the amplitude tune-spread occurs off-diagonally.

No evidence particles are bound by the resonance lines. 


\section{Higher Periodicity Lattice, Higher charge}




\section{iRCS v2 Lattice Parameters}

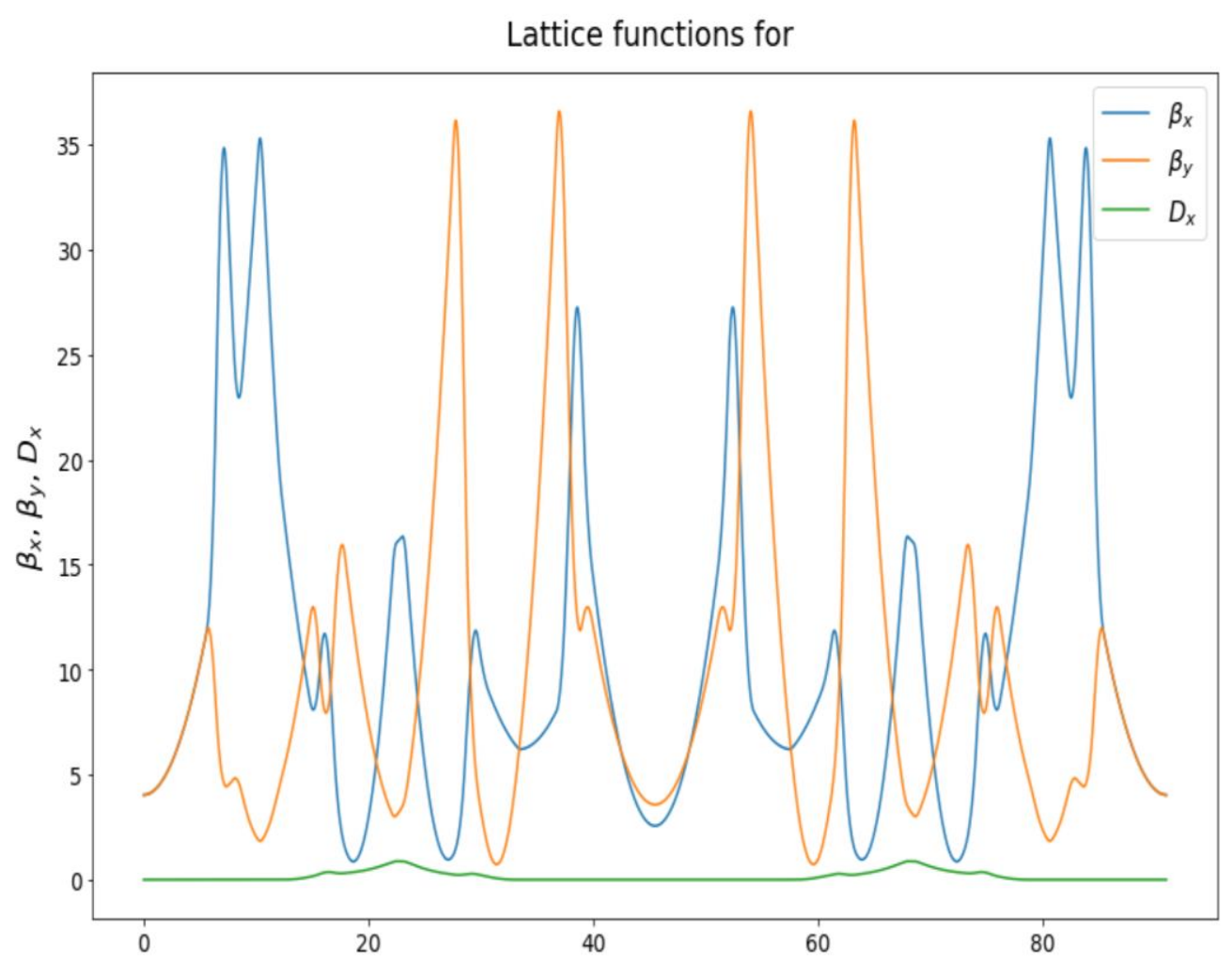

Periodicity: 6

Circumference: $\mathbf{5 4 2} \mathbf{~ m}$ Bend-radius rho: $15.4 \mathrm{~m}$ Max Beta function: $35 \mathrm{~m}$ Insertion length: $\mathbf{1 1 . 2} \mathbf{~ m}$ Betatron Tune: $\mathbf{1 6 . 8}$

Insert Phase-Advance: $\mathbf{0 . 3}$

Nonlinear t-value: $\mathbf{0 . 1 5}$ Minimum c-value: $3 \mathrm{~cm}$ Beta at insert center: $\mathbf{4} \mathbf{~ m}$ Corrected Chromaticity: $\mathbf{- 7 . 7}$ Natural Chromaticity: $\mathbf{- 3 3}$ Second-order Chromaticity: -132

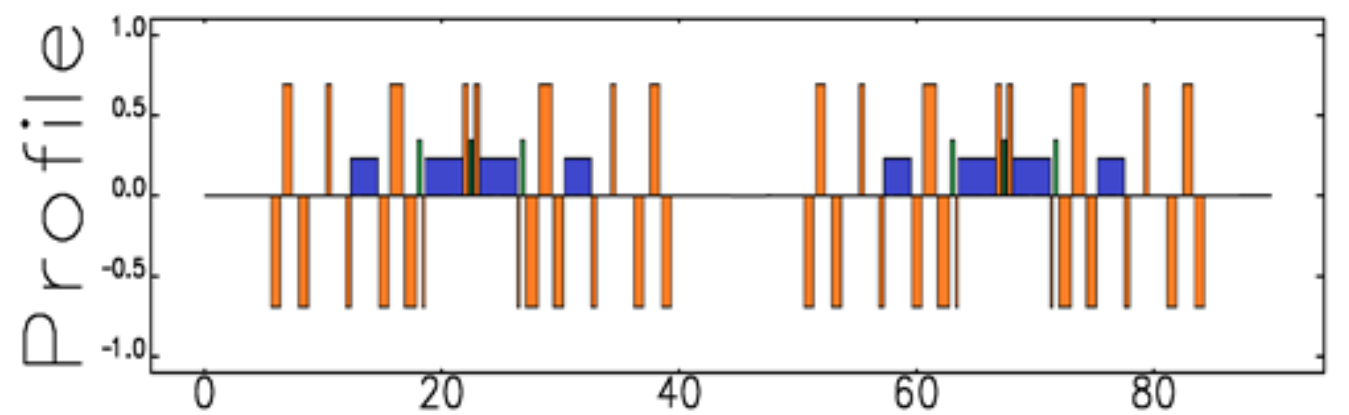




\section{iRCS v3 Lattice Parameters}
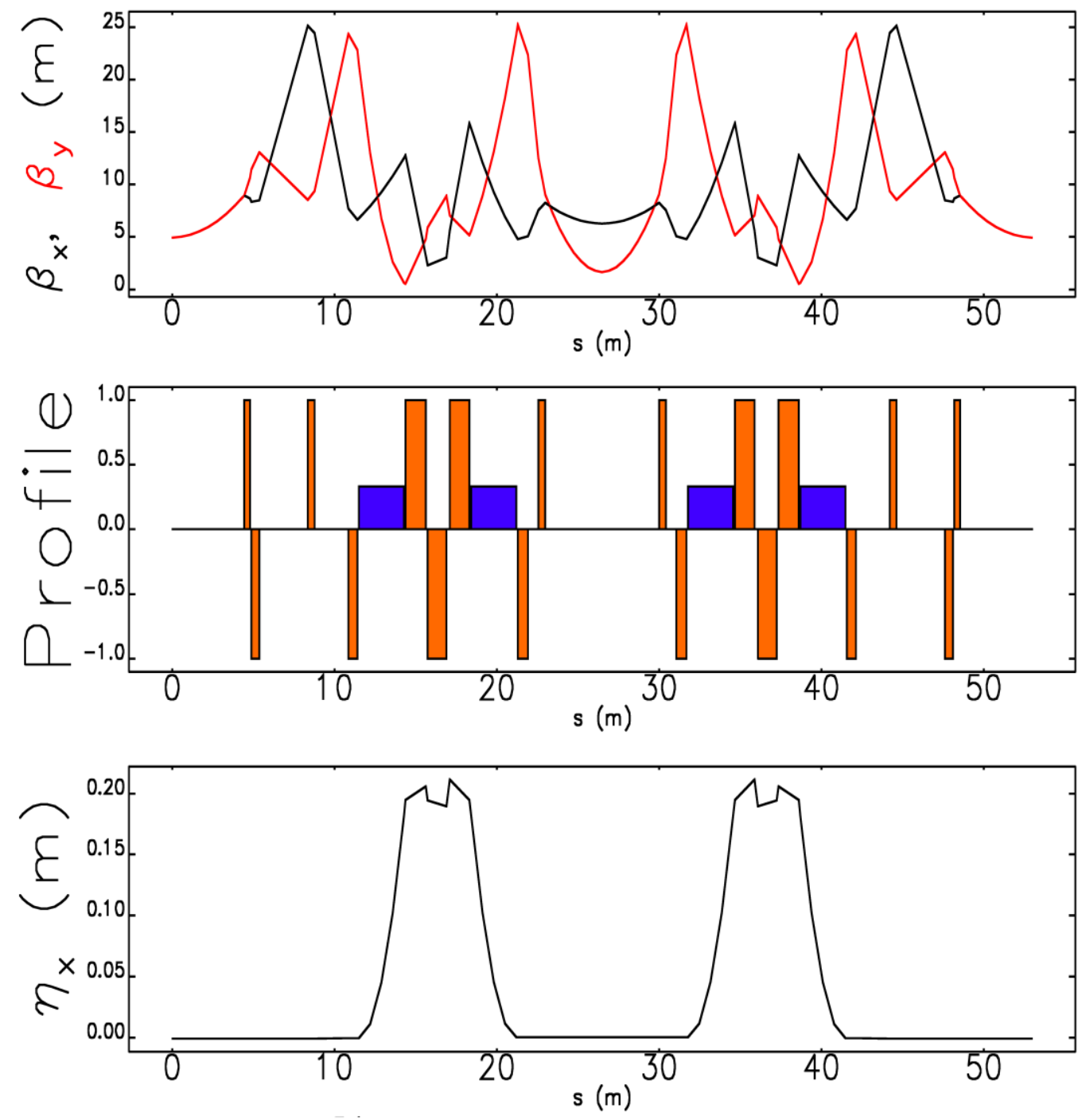

Periodicity: 12

Circumference: $636 \mathrm{~m}$ Bend-radius rho: $15.4 \mathrm{~m}$ Max Beta x,y function: 25 m Max Dispersion function: $\mathbf{0 . 2 2} \mathrm{m}$

RF Insertion length: $7.2 \mathrm{~m}, \mathbf{2 x} \mathbf{1 . 4 m}$ $\mathrm{NL}$ Insertion length: $12 \mathrm{~m}$

Insert Phase-Advance: $\mathbf{0 . 3}$ Minimum c-value: $\mathbf{3} \mathbf{~ c m}$ Beta at insert center: $\mathbf{2 . 2}$ m

Betatron Tune: $\mathbf{2 1 . 6}$ Natural Chromaticity: $\mathbf{- 7 4}$ 


\section{Cell Betatron Tune Distribution}

4 Periodicity 6, $d Q=0.2$

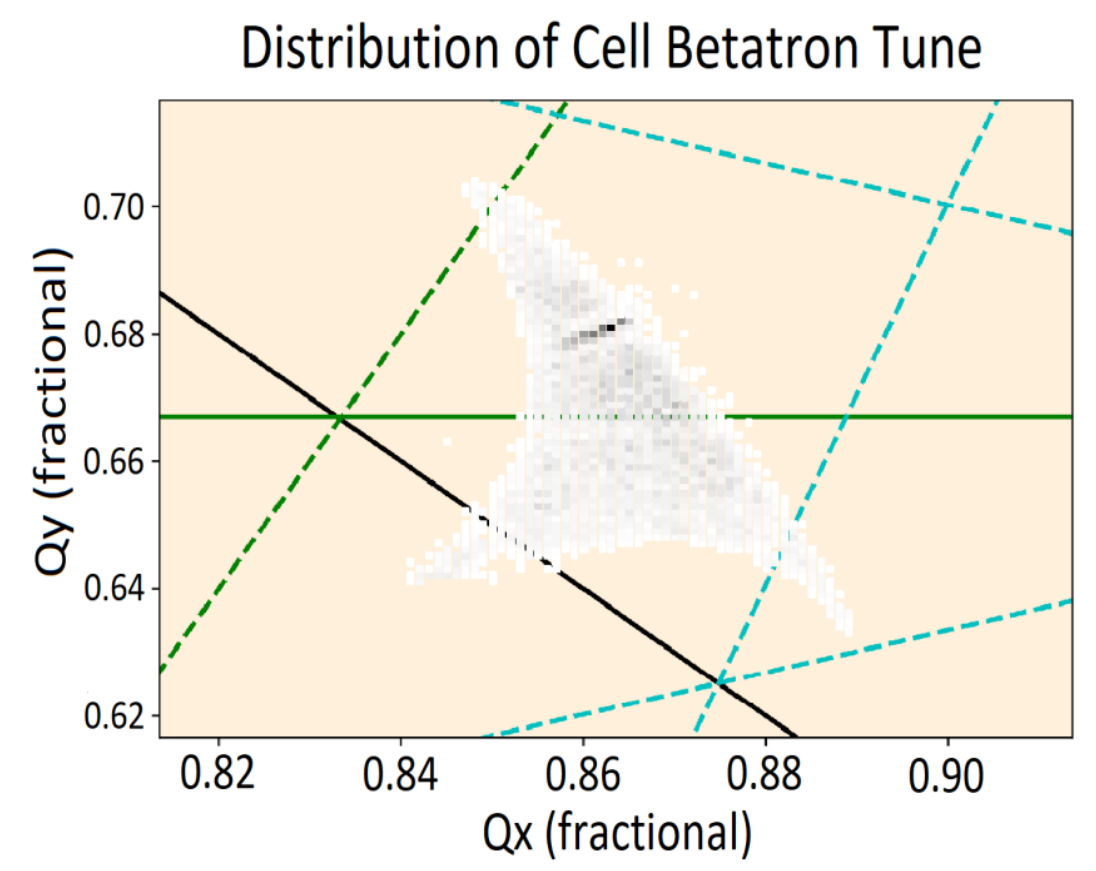

6 Periodicity 12, dQ $=0.4$

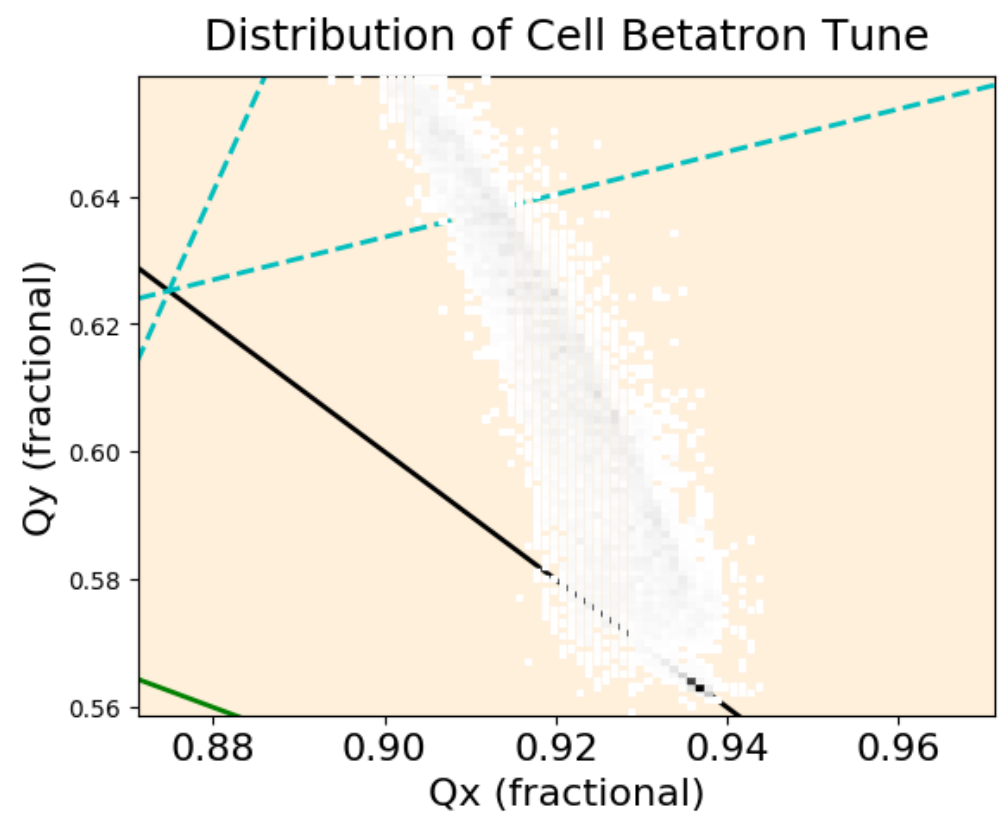




\section{RMS Beam Size}

4 Periodicity 6, dQ $=0.2$

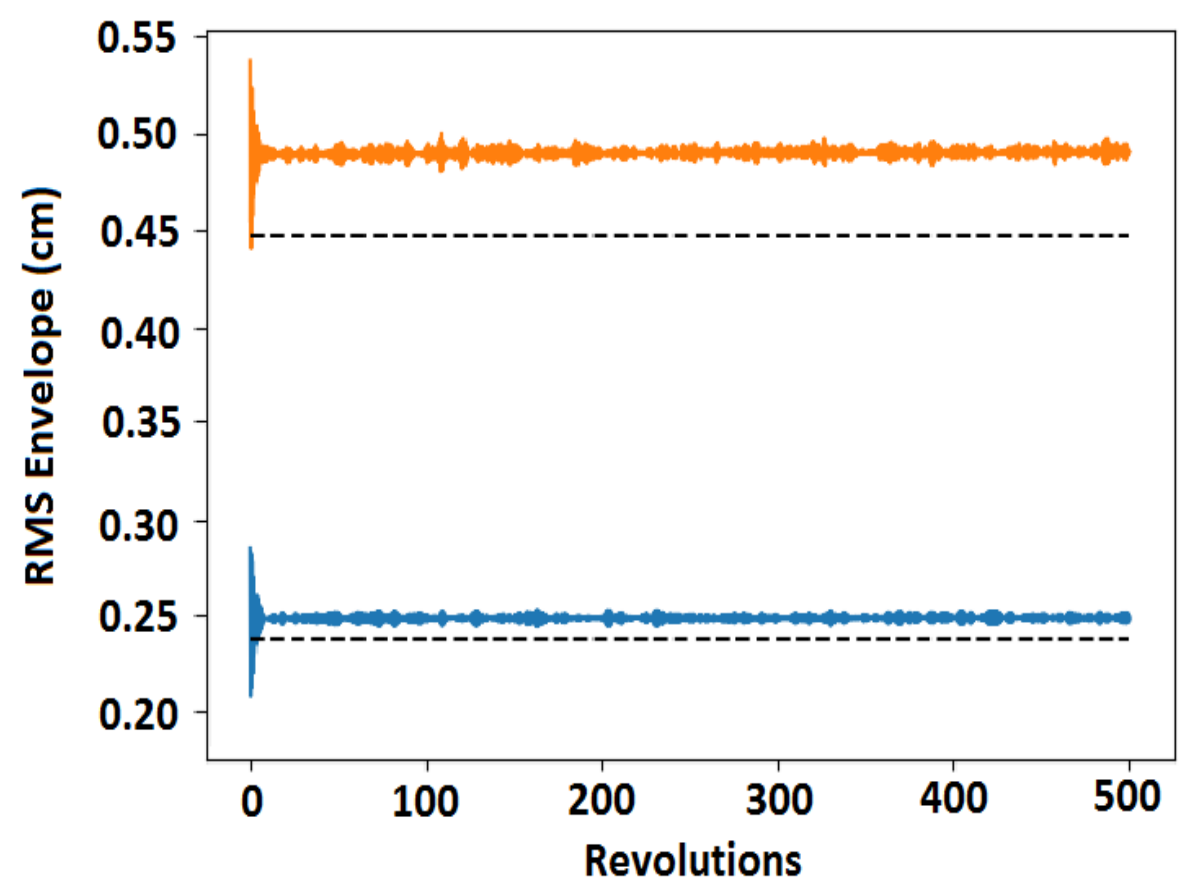

6 Periodicity $12, \mathrm{dQ}=0.4$

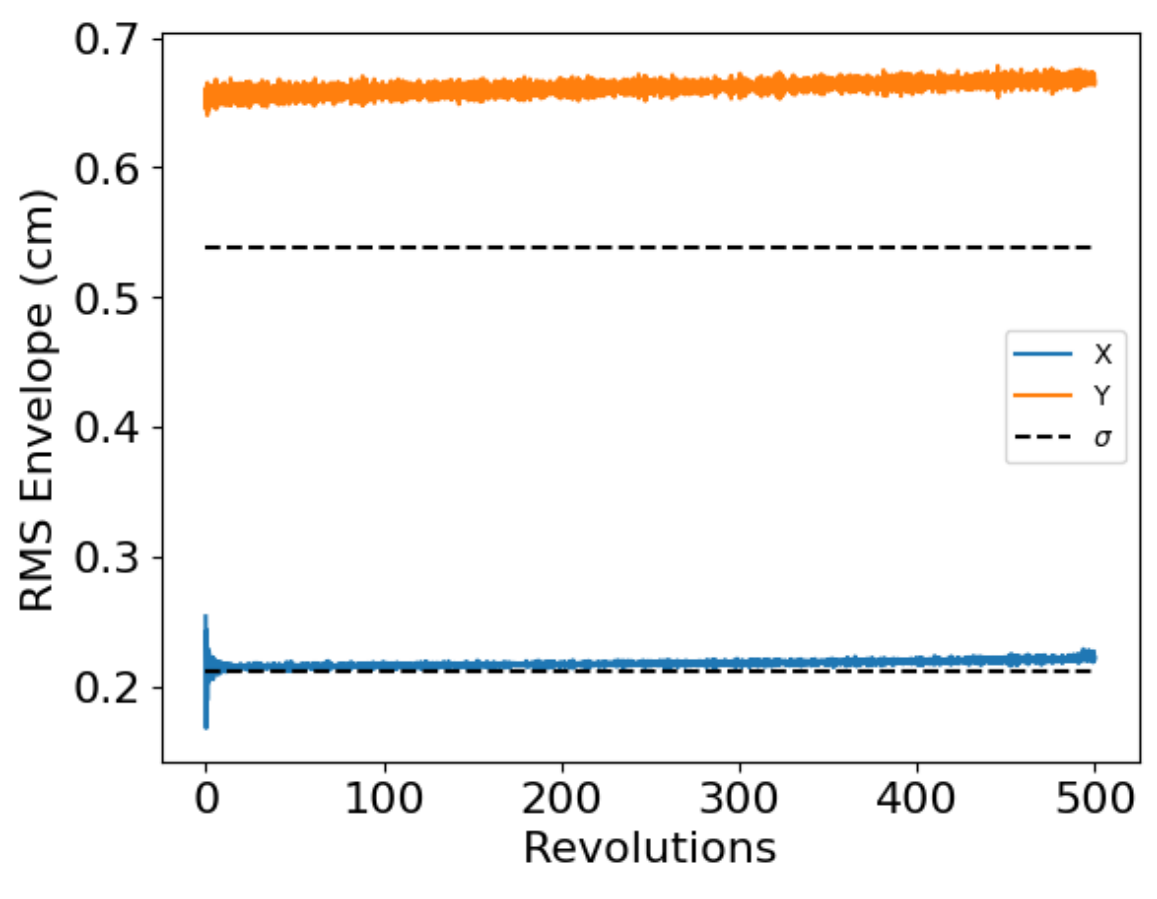




\section{Transverse Beam Halo}

4 Periodicity 6, dQ $=0.2$

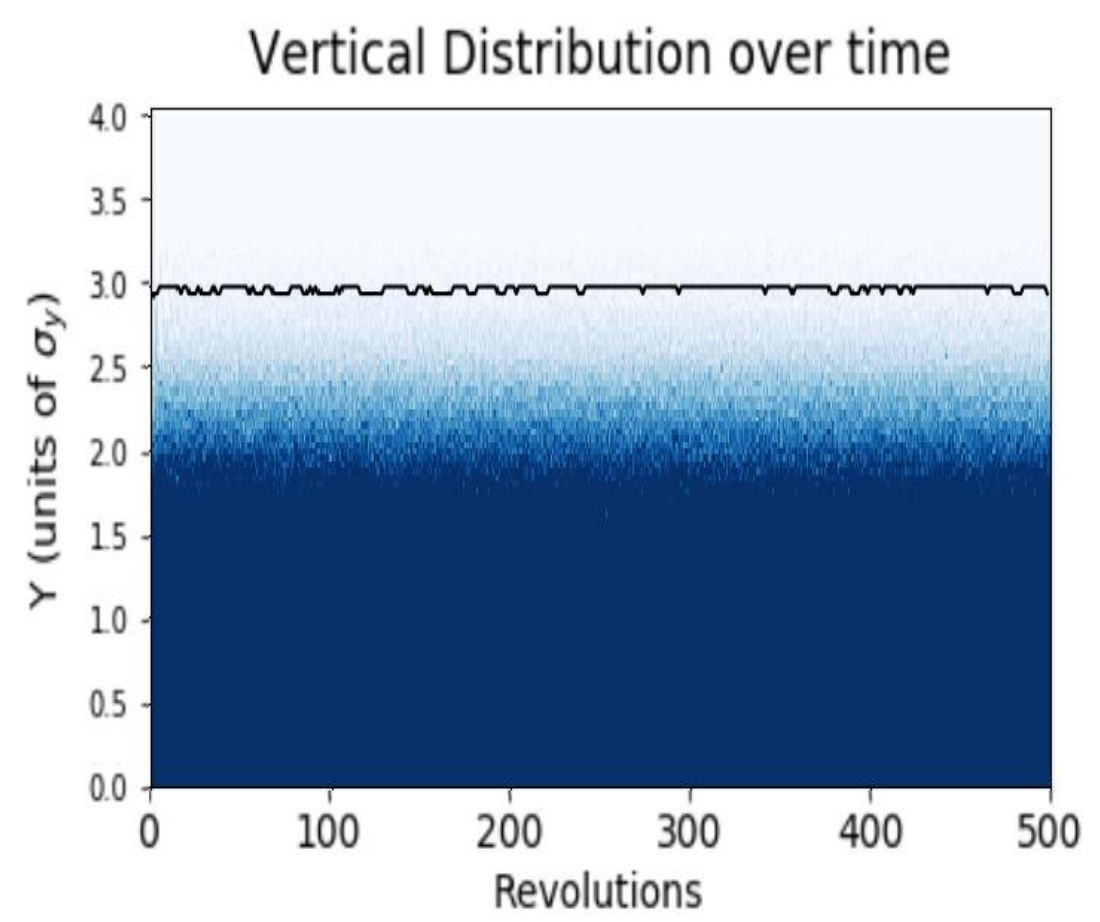

6 Periodicity $12, \mathrm{dQ}=0.4$

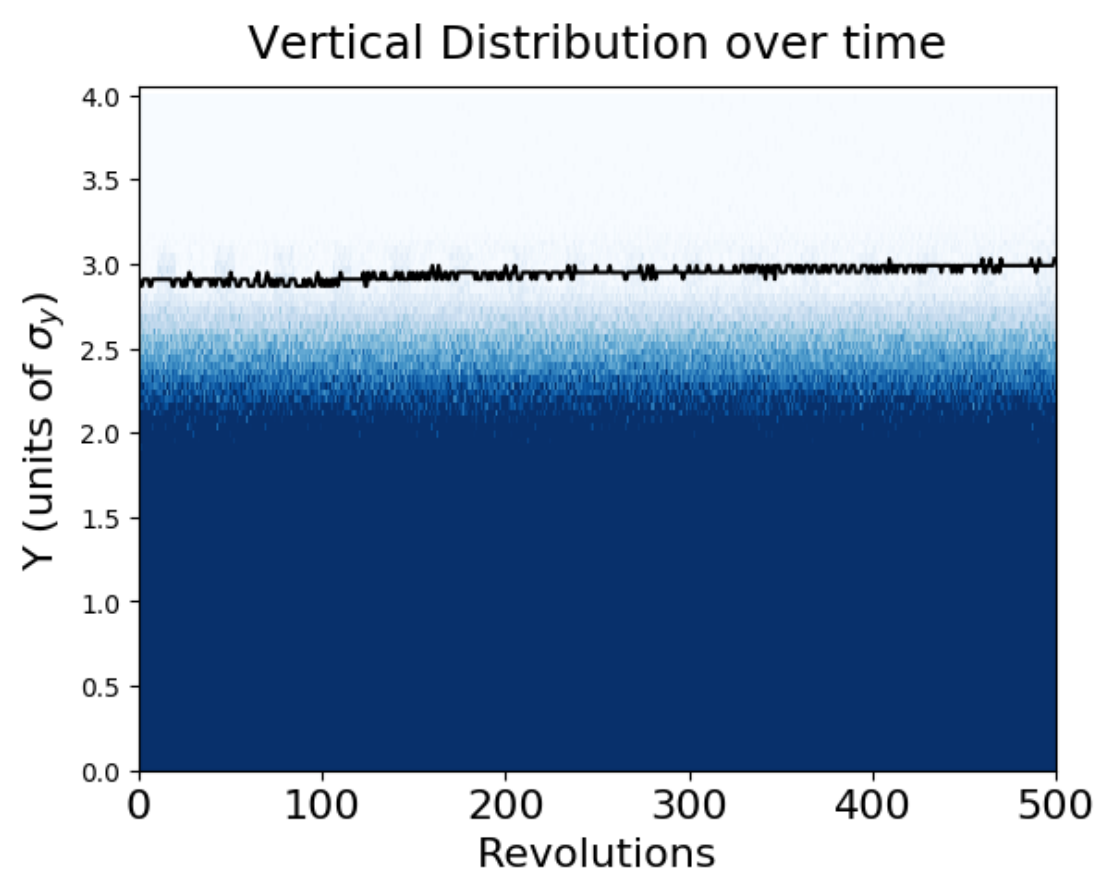




\section{Summary \& Upcoming Work}

\section{Summary:}

- Integrable optics provide a large nonlinear tune-spread without introducing new resonances and can be used to mitigate the formation of beam halo.

- Integrable optics is reasonably tolerant to space-charge and chromatic phase-errors.

- New lattice will examine advantages of high periodicity.

\section{Upcoming Work:}

- Analyze particles trajectories to better understand the physics.

- Use random quad errors to break periodicity and investigate effects of integrable optics.

- Bunched beam with RF and 3D space-charge forces. 